\title{
A learned flavor preference persists despite the extinction of conditioned hedonic reactions to the cue flavors
}

\author{
DOMINIC M. DWYER \\ Cardiff University, Cardiff, Wales \\ AND \\ Hannah L. Pincham, Thida Thein, and Justin A. Harris \\ University of Sydney, Sydney, New South Wales, Australia
}

\begin{abstract}
Learned flavor preferences can be strikingly persistent in the face of behavioral extinction. Harris, Shand, Carroll, and Westbrook (2004) suggested that this persistence may be due to flavor preference conditioning's producing a long-lasting change in the hedonic response to the conditioned stimulus $(\mathrm{CS}+)$ flavor. In the present study, the CS + flavor was presented in simultaneous compound with $16 \%$ sucrose, whereas the CS - flavor was presented with $2 \%$ sucrose. During subsequent two- and one-bottle tests, the CS + and CS - flavors were presented in $2 \%$ sucrose. Hedonic reactions during training and test were assessed using an analysis of the microstructure of licking behavior. Conditioning resulted in greater consumption of the CS + than of the CS - that did not extinguish over repeated two- and one-bottle tests. The mean lick cluster size was higher for the CS + than for the CS - only on the first cycle of tests. Since lick cluster size can be used as an index of stimulus palatability, the present results indicate that although the hedonic reaction to the CSs did change, this was not maintained across repeated tests. Thus, changes in the hedonic response to the conditioned flavors cannot explain the resistance to the extinction of learned flavor preferences.
\end{abstract}

Learning plays a major role in food selection and preference for omnivorous animals, such as rats or humans. One example of such learning is flavor preference conditioning, in which animals can learn to prefer flavors that are associated with positive consequences. Methods for conditioning flavor preferences include flavor-nutrient learning, in which the conditioned stimulus (CS) flavor is paired with nutrients, such as starch (see, e.g., Sclafani \& Nissenbaum, 1988), as well as flavor-flavor learning, in which the CS is paired with the oral consumption of a particularly palatable taste, such as saccharin (e.g., Holman, 1975). These conditioned preferences can be remarkably persistent even when the positive consequences on which the preference was originally based are no longer present (e.g., Capaldi, Myers, Campbell, \& Sheffer, 1983; Drucker, Ackroff, \& Sclafani, 1994; Harris, Shand, Carroll, \& Westbrook, 2004).

It should be noted that the removal of the unconditioned stimulus (US) is not without effect in flavor preference conditioning. Delamater (2007) showed that rats will consume a nonextinguished $\mathrm{CS}+$ in preference to an extinguished CS + . There was no difference, however, between the extinguished and nonextinguished $\mathrm{CS}+\mathrm{s}$ in terms of their preferential consumption when pitted against a $\mathrm{CS}-$. Thus, there is evidence for resistance to some aspects of behavioral extinction. Furthermore, Harris et al. (2004) demonstrated that after training a preference for a CS by pairing it with sucrose, presenting the CS alone prevented a subsequent devaluation of sucrose from influencing CS consumption. Critically, the same CS-alone presentations did not affect the preference for the CS over water. In order to account for this pattern of results, Harris et al. suggested that consuming the CS in compound with sucrose served to pair the CS with the hedonic reaction elicited by sucrose, which, in turn, resulted in the CS's having the same hedonic properties as sucrose. These acquired hedonic properties of the CS should maintain the preference independently of the flavor-sucrose association.

The account suggested by Harris et al. (2004) assumes that preference conditioning will result in a change in the palatability of the CS flavors, and there is good evidence that such changes do take place. In particular, taste reactivity testing has been used to show that preferences that are based on intragastric infusions of nutrients or the oral consumption of calorie-rich substances are associated with an increase in appetitive reactions to the CS (Forestell \& LoLordo, 2003; Myers \& Sclafani, 2001b). These conditioned changes in the palatability of the CS are not simply a reflection of the increased preference for the $\mathrm{CS}+$ after conditioning, because using saccharin as the US allowed

D. M. Dwyer, dwyerdm@cardiff.ac.uk 
for preference conditioning to take place in the absence of any modification of the hedonic reactions to the CS+ (Forestell \& LoLordo, 2003). In addition, using aversive tastes (e.g., bitter or sour) as cues prevented any conditioned changes in the hedonic reaction to the CSs but did not prevent a conditioned preference for the CS + developing (Myers \& Sclafani, 2003). Thus, conditioned changes in CS consumption can occur independently of conditioned changes in the hedonic reaction to the CSs.

Although the taste reactivity test has been the most commonly used method for investigating palatability changes produced by taste-aversion learning, the technique does have a number of pragmatic problems. For example, taste reactivity analyses are normally performed on the handscored responses of animals to the passive infusion of fluids directly into their oral cavity. Doing this is time consuming and potentially introduces a context change if the training sessions are not also based on passive infusion. In addition, taste reactivity analyses are normally limited to a small sample of behavior. Analyzing the microstructure of licking behavior provides an alternative assay of animals' hedonic reaction to flavors that is not subject to the same pragmatic constraints as is taste reactivity testing. When ingesting fluids, rats produce sustained runs of rapidly occurring rhythmic licks (referred to here as "clusters") that are separated by pauses of varying length. The mean number of licks in a cluster bears a positive, monotonic relationship to the concentration of palatable fluids, which has led to the suggestion that cluster size is an index of stimulus palatability (see, e.g., Davis \& Smith, 1992; Spector, Klumpp, \& Kaplan, 1998). Importantly, cluster size does not simply reflect the amount of solution consumed, since there is typically an inverted U-shaped function between concentration and total consumption, with the highest levels of consumption at intermediate concentrations of palatable solutions. Therefore, cluster size is independent of consumption level. With regard to aversive tastes, cluster size decreases monotonically with increasing concentrations of unpalatable quinine solutions (e.g., Hsiao \& Fan, 1993), and conditioned taste aversions are also accompanied by reductions in cluster size (e.g., Dwyer, 2009; Dwyer, Boakes, \& Hayward, 2008). The assumption that cluster size directly reflects palatability receives further support from the fact that pharmacological treatments that are thought to influence palatability also have a direct effect on cluster size (e.g., Higgs \& Cooper, 1998). The analysis of the licking microstructure has also been applied to acquired preferences (Dwyer, 2008; Myers \& Sclafani, 2001a) in which conditioned preferences are associated with increases in lick cluster size (analogous to raising the concentration of a palatable substance).

The fact that flavor preference learning can produce an increase in the palatability of the CS + is exactly the state of affairs assumed by Harris et al. (2004) as the basis for their suggestion that the persistence of conditioned flavor preferences is due to the acquisition of a heightened hedonic reaction to the $\mathrm{CS}+$. However, previous studies of acquired palatability in flavor preference learning have not examined whether changes in CS palatability are maintained over repeated tests. So, although both taste reactivity and lick-analysis methods indicate that flavor preference learning can produce changes in CS palatability, it is not known whether these changes persist over repeated testing. Thus, the present study analyzed lick microstructure to assess whether or not persistent changes in CS palatability can account for the apparent resistance to the extinction of flavor preference learning.

\section{METHOD}

\section{Subjects and Apparatus}

Thirty-two experimentally naive male hooded Wistar rats that were obtained from the University of Sydney breeding program were used. Their weights were in the range of 169 to $303 \mathrm{~g}$ at the start of the experiment. All were housed in groups of 8 in a colony room under a 12:12-h light:dark cycle. Animals were maintained on ad lib access to food and water throughout the experiment.

Training and testing took place in a room that contained 16 custommade automated drinking chambers measuring $30 \times 19 \times 18 \mathrm{~cm}$ high. The floors were steel mesh; the side walls were steel sheet; the rear wall was a steel access door; and the front wall was made of vertical steel rods. The front wall was fitted with a Plexiglas sheet with two $1-\mathrm{cm}$-diameter holes that were approximately $8 \mathrm{~cm}$ above the cage floor and $2.5 \mathrm{~cm}$ to either side of the midline of the front wall. Fluids could be made available through stainless steel drinking spouts from 120-ml plastic bottles (Rolf C. Hagen Inc.) that were mounted so that the spouts were level with the inside edge of the Plexiglas cover sheet. A contact-sensitive lickometer registered the time of each lick to the nearest $0.001 \mathrm{sec}$, and a computer recorded the data. The CS solutions were $1 \%(\mathrm{v} / \mathrm{v})$ almond and $2 \%(\mathrm{v} / \mathrm{v})$ rose essence (Queen, Australia). The identity of the CS + and CS - flavors was counterbalanced across animals. During training, the CS + flavor was presented in simultaneous compound with $16 \%$ sucrose (CSR, Australia), and the CS - flavor was presented in simultaneous compound with $2 \%$ sucrose. During testing, both the CS + and CSwere presented in compound with $2 \%$ sucrose.

We added $2 \%$ sucrose to the $\mathrm{CS}-$ solution during training and to the $\mathrm{CS}+$ and $\mathrm{CS}-$ solutions during test, to ensure their consumption by the nondeprived rats. We chose this procedure on the basis of previous studies in which we presented unsweetened CS - and CS + solutions to water-deprived rats during the test sessions. Those previous experiments revealed that water deprivation tended to drive cluster size toward a ceiling, thus masking more subtle differences in palatability.

\section{Procedure}

Animals were initially trained to consume fluids in the experimental chambers by giving them access to $2 \%$ sucrose over daily 15 -min sessions. After two pretraining sessions, conditioning began. This consisted of six sessions with free access to the CS + flavor with 16\% sucrose and six sessions with free access to the CS - flavor combined with $2 \%$ sucrose. The duration of these sessions differed (10 min for $\mathrm{CS}+$ and $15 \mathrm{~min}$ for $\mathrm{CS}-$ ) in order to minimize differences in the total intake of the two solutions (because we anticipated that the rats would otherwise drink more of $\mathrm{CS}+$ than of $\mathrm{CS}-$ ). Animals received one session per day, and CS + and CS - sessions were given in double alternation (half of the animals began with a CS + session, and the remainder with the CS-). The position of the bottles (on the left vs. on the right of the front wall of the drinking chamber) alternated across days, so that each solution (CS + and $\mathrm{CS}-$ ) occurred equally often on the left and right. After this training was completed, animals received three 3-day test cycles. Each cycle began with a one-bottle test, with either the CS + or CS - flavor in $2 \%$ sucrose. This was followed $24 \mathrm{~h}$ later by a second one-bottle test with the other flavor $(\mathrm{CS}-$ or $\mathrm{CS}+)$ in $2 \%$ sucrose. The order of exposure to the CS+ and CS - was counterbalanced across animals. The position of the bottles alternated across the two one-bottle tests, but the allocation of solution $(\mathrm{CS}+$ vs. $\mathrm{CS}-)$ to position was counterbalanced both between rats and across test cycles. On the third day of the cycle, the rats received a two-bottle test with the $\mathrm{CS}+$ and $\mathrm{CS}-$ flavors 
presented simultaneously (the side on which the $\mathrm{CS}+$ and the $\mathrm{CS}-$ was presented was counterbalanced across animals and across tests). All one- and two-bottle tests sessions were $15 \mathrm{~min}$ in length.

\section{Data Analysis}

Consumption was assessed by weighing the drinking bottles before and after each session. The mean cluster size (i.e., the mean number of licks in the clusters recorded in a single test session for each animal) was extracted from the record of licks, with a cluster being defined as a set of licks, where each lick is separated by an interlick interval of no more than $1 \mathrm{sec}$. This criterion was adopted by Spector et al. (1998) to define licking, and although other criteria have been used (e.g., $0.5 \mathrm{sec}$ by Davis \& Smith, 1992), parametric analyses suggest that there is little practical difference between them, since most pauses greater than $0.5 \mathrm{sec}$ are also greater than $1 \mathrm{sec}$ (see, e.g., Davis \& Smith, 1992; Spector et al., 1998). We analyzed the present data using both criteria, and although estimated bout lengths were slightly shorter with the 0.5 -sec criterion, the pattern of results was virtually identical to that reported below using the 1-sec criterion. Lick analysis will be reported only for the one-bottle sessions, because the opportunity to switch between drinking bottles tends to disturb the pattern of licking in two-bottle tests. Three animals were removed from the analysis, 1 because it died before the experiment was completed, and 2 because equipment failure led to incomplete lick recording in at least one of the six one-bottle test sessions. Because the CS + and CS - training sessions were of different lengths, the analysis of licking behavior was restricted to the first $10 \mathrm{~min}$ of the $\mathrm{CS}$ - training sessions.

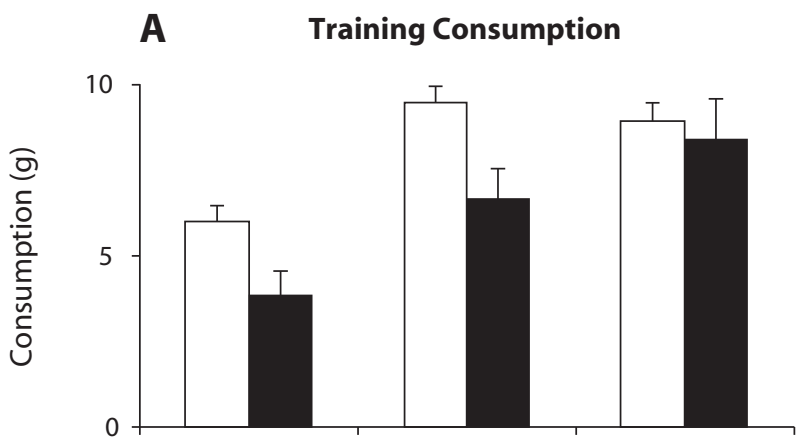

B Training Palatability

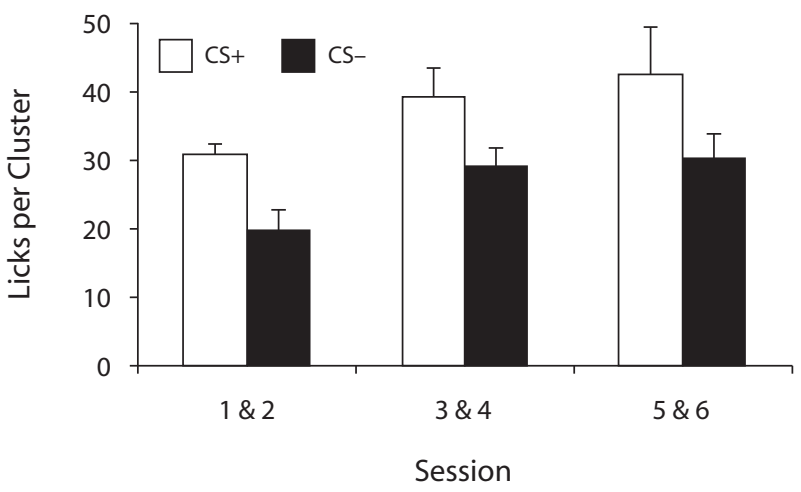

Figure 1. Training phase data as a factor of conditioned stimulus (CS) type and session (note that bars represent two-session blocks). During training, the CS+ was presented in simultaneous compound with $16 \%$ sucrose, and the CS- with $2 \%$ sucrose. (A) Mean consumption (with $S E M$ ) of the CS+ and CS- flavored solutions. (B) Mean number of licks per cluster (with the $S E M$ ) during the consumption of the $\mathrm{CS}+$ and $\mathrm{CS}-$.

\section{RESULTS}

Figure 1 shows data for both consumption ( panel A) and lick cluster size (panel B) across CS + and CS - training sessions (averaged in two-session blocks). With respect to consumption, an ANOVA revealed that the effect of CS type did not quite reach the standard level of significance $[F(1,28)=4.20, p=.050]$ and that there was a significant effect of training block $[F(2,56)=44.34, p<.001]$. There was an interaction between these factors $[F(2,56)=$ $4.13, p=.001]$, and simple main effects revealed that the difference between CS + and CS - consumption was significant in the first two training blocks $[F(1,28)=14.90$, $p=.001$, and $F(1,28)=7.72, p=.010$, respectively], but this was not significant in the final block of training $(F<1)$. Therefore, giving the rats more time to consume the CS - than the CS + partially equalized consumption, despite the presence of $16 \%$ sucrose in compound with the CS+, especially at the end of training. With respect to lick cluster size, an ANOVA revealed significant effects of CS type $[F(1,28)=14.51, p=.001]$ and training block $[F(2,56)=5.80, p=.005]$. There was no interaction between these factors $(F<1)$. That larger lick cluster sizes were produced when rats consumed the CS + and US compound confirms that the lick analysis technique is sensitive to palatability differences based on different sucrose concentrations.

The most important data come from the three test cycles in which both the CS + and CS - flavors were presented in the same weak concentration of sucrose (2\%). Panel A of Figure 2 indicates that the rats showed a clear preference for the CS+ over the CS - when allowed to choose between them in two-bottle tests. A within-subjects ANOVA revealed effects of CS type $[F(1,28)=21.02, p<.001]$ and test cycle $[F(2,56)=5.72, p=.005]$. Simple effects analyses revealed that overall consumption was greater in Cycle 2 than in either Cycle 1 or $3[F(1,27)=15.034$, $p=.001$, and $F(1,27)=5.38, p=.028$, respectively], which did not differ from each other $(F<1)$. Crucially, there was no interaction between these factors $(F<1)$, indicating that the size of the CS + preference did not change over testing. Figure 2 also shows the preference ratio for the $\mathrm{CS}+$, calculated as (CS+ consumption)/ $[(\mathrm{CS}+$ consumption $)+(\mathrm{CS}-$ consumption $)]$. An analysis of this ratio data revealed no effect of test cycle $[F(2,56)=$ $1.56, p=.219]$, which confirms that the CS + preference did not extinguish over repeated testing.

Panel B of Figure 2 shows consumption during onebottle testing. An ANOVA revealed effects of CS type $[F(1,28)=11.83, p=.002]$ and test cycle $[F(2,56)=$ $43.82, p<.001]$. Simple-effects analyses revealed that overall consumption was lower in Cycle 1 than in Cycle 2 $[F(1,28)=59.17, p<.001]$, which was, in turn, lower than that in Cycle $3[F(1,28)=7.77, p=.009]$. There was an interaction between these factors $[F(2,56)=8.14$, $p=.001]$, and simple main effects revealed that the difference between CS + and CS - consumption did not reach the standard level of significance in Cycle $1[F(1,28)=$ $2.04, p=.164]$, but it was significant in Cycles 2 and 3 $[F(1,28)=13.45, p=.001$, and $F(1,28)=12.96, p=$ 

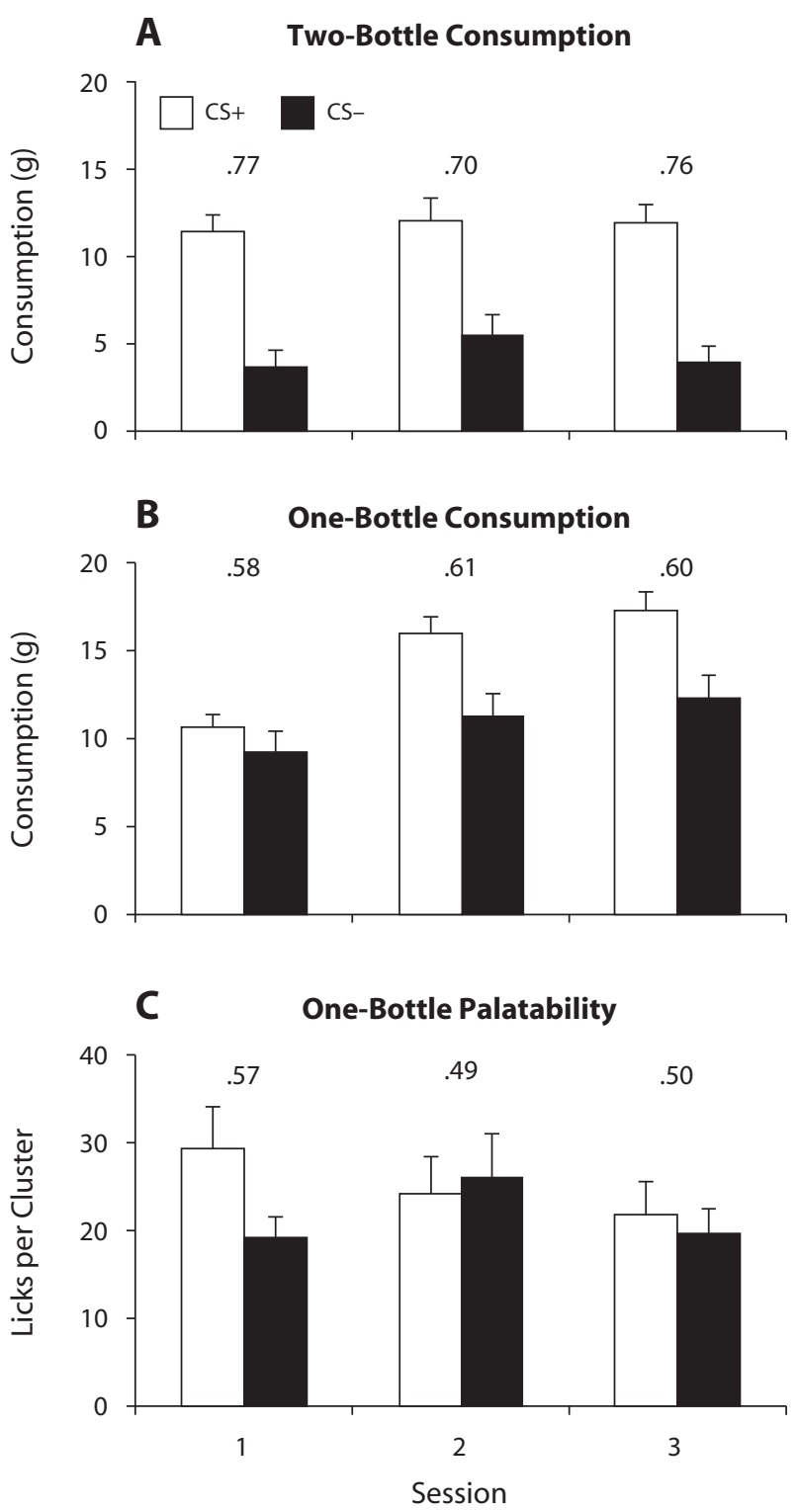

Figure 2. Test phase data as a function of conditioned stimulus (CS) type and test cycle. Both the CS+ and CS- were presented in simultaneous compound with $2 \%$ sucrose during testing. (A) Mean consumption (with the SEM) of the CS+ and CSflavored solutions in two-bottle tests. (B) Mean consumption (with the $S E M$ ) of the CS+ and CS- flavors in one-bottle tests. (C) Mean number of licks per cluster (with the $S E M$ ) during consumption of the CS+ and CS- flavors in one-bottle tests. Numbers adjacent to each pair of bars represent the ratio of responding to the $\mathrm{CS}+$ to total responding.

.001 , respectively]. It is worth noting that the lack of a significant difference between CS + and CS - consumption in the first test cycle might not be a true reflection of the relative preference for the $\mathrm{CS}+$ and $\mathrm{CS}-$ at this time: Two rats showed abnormally high consumption per lick on the initial CS - test, which is indicative of a possible bottle leak. A reanalysis of the first test cycle with these rats excluded showed a significant difference between the consumption of the CS + $(M=10.56 \mathrm{~g}, S E M=0.79)$ and the CS $-(M=8.64 \mathrm{~g}, S E M=1.14)[t(28)=2.11$, $p=.045]$. In addition, there was a significant difference in the number of licks produced for the CS + $(M=1,298$, $S E M=130)$ and $C S-(M=813, S E M=108)$ across all animals in the first test cycle $[t(28)=3.95, p<.001]$. The CS + acceptance ratio was calculated in the same way as for the two-bottle data. An ANOVA revealed no effect of test cycle $[F(2,56)=1.01, p=.372]$, and onesample $t$ tests indicated that the $\mathrm{CS}+$ acceptance ratio was higher than .5 in all test sessions $[t(28)=2.49, p=.019$; $t(28)=3.27, p=.003$; and $t(28)=3.31, p=.003$, for Sessions $1-3$, respectively]. Thus, the ratio data suggest that the lower absolute difference between CS + and CSconsumption in Cycle 1 was at least partially due to lower overall levels of consumption, as opposed to reflecting a lack of preference per se.

Finally, panel C of Figure 2 shows the mean cluster size during one-bottle testing. An ANOVA revealed no significant effect of either CS type $[F(1,28)=3.16, p=.086]$ or test cycle $[F(2,56)=1.80, p=.175]$; however, the interaction between these factors was significant $[F(2,56)=$ $3.19, p=.049]$. Simple main effects of this interaction revealed that the difference between the $\mathrm{CS}+$ and the CS - cluster size was significant in Cycle $1[F(1,28)=$ $10.13, p=.004]$, but not in Cycles 2 and $3\left(F_{\mathrm{s}}<1\right)$. This pattern of results indicates that, although conditioning initially produced a difference in the palatability of the $\mathrm{CS}+$ and the $\mathrm{CS}-$, this was not maintained over repeated testing. This interpretation is consistent with the analysis of the palatability ratio for the $\mathrm{CS}+$ (calculated using the $\mathrm{CS}+$ and the CS - cluster size and the same formula as that for consumption): An ANOVA revealed that the effect of test cycle approached standard levels of significance $[F(2,56)=3.08, p=.054]$. Although the overall ANOVA did not reach standard levels of significance, a simple-effects analysis revealed that the palatability ratio was higher in Cycle 1 than in either of Cycles 2 or 3 $[F(1,28)=5.03, p=.033$, and $F(1,28)=4.27, p=.048$, respectively]. As was noted earlier, a virtually identical pattern of results was obtained when the licking microstructure was analyzed using a 0.5 -sec criterion as the cutoff for within- versus between-cluster pauses. However, with this analysis, the interaction between CS type and test cycle fell just short of significance, even though the simple effects analyses confirmed that there was a significant difference in cluster size on Cycle 1, but not on Cycle 2 or 3.

\section{DISCUSSION}

In summary, pairing a CS + flavor with $16 \%$ sucrose resulted in a subsequent preference for that flavor when tested against a CS - flavor that had previously been paired with $2 \%$ sucrose. This preference was apparent in both two- and one-bottle tests and did not reduce, despite repeated testing in extinction. This result replicates those in previous reports that flavor preferences are resistant to behavioral extinction (see, e.g., Capaldi et al., 1983; Drucker et al., 1994; Harris et al., 2004). The novel finding from the present study is that although preference 
training initially produced a difference in the lick cluster size during consumption of the $\mathrm{CS}+$ and $\mathrm{CS}-$, this difference was not maintained across repeated testing. Thus, the persistent preferential consumption of the CS + over the CS - in extinction is not accompanied by an equally persistent change in the palatability of the CSs. This dissociation between lick cluster size and consumption measures is not consistent with the suggestion by Harris et al. that flavor preference conditioning should imbue a CS+ flavor with the same hedonic tone as that of the US.

One noteworthy observation in the present experiment is that consumption during the one-bottle tests increased over repeated test cycles. However, this increase in consumption interacted with CS type in a way that was very different from the way in which CS type interacted with cluster size: the CS + versus CS - difference in consumption was small at the first test but increased on the subsequent tests, whereas the CS + versus CS- difference in cluster size was large at the first test and absent on the subsequent tests. As was argued previously, these results are clearly inconsistent with the idea that resistance to extinction in flavor preference learning is mediated by a permanent change in the palatability of the CSs. But what can explain the overall change in the amount consumed? One possibility is that consumption changes reflect differences in adaptation level (see, e.g., Albertella, Harris, \& Boakes, 2008; Boakes, Albertella, \& Harris, 2007). Across repeated presentations of $2 \%$ and $16 \%$ sucrose during training, the rats would have adapted to an intermediate level of sweetness, which would have diminished their sensitivity to the $2 \%$ sucrose provided at a subsequent test. If the rats had readapted to this consistently lower concentration of sucrose across test cycles, their sensitivity to the $2 \%$ sucrose would have progressively increased, and with this increase in sensitivity, their intake of the test solution would have also increased.

It is possible that a successive negative contrast effect (for a review, see Flaherty, 1996) might have resulted in lowered CS + consumption on the first one-bottle test. That is, consumption of the $2 \%$ sucrose present during test might have been reduced because of the negative contrast with the $16 \%$ sucrose that had previously been presented with the CS+. Since the CS- was presented with $2 \%$ sucrose throughout, any such contrast would have been reduced in these tests. If so, then the same effect should have also attenuated the positive hedonic reaction to the $2 \%$ sucrose during CS + testing, since successive negative contrast has also been shown to reduce lick cluster size (Grigson, Spector, \& Norgren, 1993). Although such a negative contrast effect is logically possible, we are unaware of any direct evidence for negative contrast effects in flavor preference procedures more generally. As was noted in the Results section, the lack of a CS + versus $\mathrm{CS}$ - difference on the first test cycle might be more apparent than it is real, and the interaction between CS+ and CS - consumption and test cycle might be a simple product of overall consumption; that is, there was a true $\mathrm{CS}+$ versus $\mathrm{CS}-$ difference throughout (consistent with the $\mathrm{CS}+$ acceptance ratio data and the number of licks for the $\mathrm{CS}+$ and $\mathrm{CS}-$ ), but the absolute size of this difference was a function of the overall amount consumed.

If long-lasting changes in the hedonic reaction to the CS flavors do not account for the observed resistance to behavioral extinction in flavor preferences, the question remains as to what mechanism(s) might do so. There is certainly a growing consensus that extinction does not remove CS-US associations but, instead, lays down new CS-No-US associations (see, e.g., Bouton, 2004; Delamater, 2004), so the observation of resistance to extinction in flavor preference could be interpreted as further evidence for this view. Interestingly, conditioned taste aversions produce a reduction in palatability (measured using either taste reactivity or lick analysis methods), which tends to extinguish before the reduction in consumption (e.g., Cantora, Lopez, Aguado, Rana, \& Parker, 2006; Dwyer, 2009). We have recently interpreted this divergence between consumption and palatability measures in taste aversion as evidence that the former are more sensitive to residual CS-US associations than are the latter (Dwyer, 2009). In brief, the argument was made that the absence of an expected US should act to minimize the expression of CS-US associations and that the nonoccurrence of the US should be particularly salient during actual consumption, thus leading to a preferential expression of CS-no-US associations over CS-US associations. During preparatory responding (including approach to the $\mathrm{CS}$ ), the absence of the US would be less salient, since its absence is not immediately apparent. The amount consumed reflects both the number of times the spout is approached (a preparatory response) and the amount consumed after each approach, whereas hedonic measures (including cluster size) reflect only consummatory responses. Therefore, if preparatory responding supports greater expression of the original CS-US association than does consummatory responding, then the original CS-US association should also have proportionally more influence on consumption than on palatability measures. A complementary possibility that is quite closely related to that suggested by Harris et al. (2004) is that the CS flavor might become able to elicit the consummatory or approach responses initially produced by the US. That is, instead of acquiring the hedonic tone of the US, the CS+ acquires the ability to promote the approach that was previously elicited by the US. This acquired tendency for the rats to approach the CS + should not extinguish, because the CS + was tested in the presence of the mildly palatable $2 \%$ sucrose and dilute sucrose has been an effective reinforcer in flavor preference learning (e.g., Dwyer, 2005).

As was noted in the introduction, flavor preferences can be created in a variety of ways (e.g., pairing a neutral CS with either palatable tastes or nutrients). In the present experiment, giving the CS-US parings as a simultaneous compound meant that the CS was paired with both the palatable taste of sucrose and the nutrients it contained. Thus, both the preference for the CS+ over the CS - and its resistance to extinction could have been the result of either CS-taste or CS-nutrient associations (or both). Because both CS-taste and CS-nutrient associations were possible 
in the present design, the present results do not speak to the issue of whether nutrient- or palatability-based preferences differ with respect to extinction. Similarly, the present results do not indicate whether the observed dissociation between conditioned palatability and conditioned preferences with respect to extinction is specific to either nutrient- or palatability-based preferences. Indeed, it is possible that the transient change in CS palatability was due to CS-nutrient associations, whereas the enduring preference for the CS+ was due to CS-taste associations. Indeed, using aversive stimuli as CSs appears to prevent conditioned changes in CS palatability despite the presence of robust preferences (Myers \& Sclafani, 2003), so the idea that conditioned preference and palatability might dissociate is not unprecedented (albeit that there is no indication that preferences conditioned to aversive CSs are also resistant to extinction).

In conclusion, we have demonstrated that although flavor preference conditioning can change the palatability of the CS flavors, this change cannot explain the persistent preference for the $\mathrm{CS}+$ over the $\mathrm{CS}-$, because consumption changes are more resistant to extinction than are palatability changes. This result parallels recent data from taste aversion learning and is consistent with the idea that consumption tests are particularly sensitive to residual CS-US associations.

\section{AUTHOR NOTE}

The present experimental work was supported by a BBSRC new investigator award and a BBSRC ISIS award to D.M.D., and by a Discovery Project grant from the Australian Research Council to J.A.H. Correspondence concerning this article should be addressed to D. M. Dwyer, School of Psychology, Cardiff University, Tower Building, Park Place, Cardiff CF10 3AT, Wales (e-mail: dwyerdm@cardiff.ac.uk).

\section{REFERENCES}

Albertella, L., Harris, J. A., \& Boakes, R. A. (2008). Acquired flavour preferences: Contextual control of adaptation-level effects. Quarterly Journal of Experimental Psychology, 61, 227-231. doi:10.1080/ 17470210701664864

Boakes, R. A., Albertella, L., \& Harris, J. A. (2007). Expression of flavor preference depends on type of test and on recent drinking history. Journal of Experimental Psychology: Animal Behavior Processes, 33, 327-338. doi:10.1080/17470210701664864

Bouton, M. E. (2004). Context and behavioral processes in extinction. Learning \& Memory, 11, 485-494. doi:10.1101//m.78804

Cantora, R., Lopez, M., Aguado, L., Rana, S., \& Parker, L. A. (2006). Extinction of a saccharin-lithium association: Assessment by consumption and taste reactivity. Learning \& Behavior, 34, 37-43.

Capaldi, E. D., Myers, D. E., Campbell, D. H., \& Sheffer, J. D. (1983). Conditioned flavor preferences based on hunger level during original flavor exposure. Animal Learning \& Behavior, 11, 107-115.

Davis, J. D., \& SмITH, G. P. (1992). Analysis of the microstructure of the rhythmic tongue movements of rats ingesting maltose and sucrose solutions. Behavioral Neuroscience, 106, 217-228.

Delamater, A. R. (2004). Experimental extinction in Pavlovian conditioning: Behavioural and neuroscience perspectives. Quarterly
Journal of Experimental Psychology, 57B, 97-132. doi:10.1080/ 02724990344000097

Delamater, A. R. (2007). Extinction of conditioned flavor preferences. Journal of Experimental Psychology: Animal Behavior Processes, 33, 160-171. doi:10.1037/0097-7403.33.2.160

Drucker, D. B., Ackroff, K., \& Sclafani, A. (1994). Nutrientconditioned flavor preference and acceptance in rats: Effects of deprivation state and nonreinforcement. Physiology \& Behavior, 56, 701-707.

DWYER, D. M. (2005). Reinforcer devaluation in palatability-based learned flavor preferences. Journal of Experimental Psychology: Animal Behavior Processes, 31, 487-492. doi:10.1037/0097-7403 .31.4.487

DWYer, D. M. (2008). Microstructural analysis of conditioned and unconditioned responses to maltodextrin. Learning \& Behavior, 36, 149-158. doi:10.3758/LB.36.2.149

DWYER, D. M. (2009). Microstructural analysis of ingestive behaviour reveals no contribution of palatability to the incomplete extinction of a conditioned taste aversion. Quarterly Journal of Experimental Psychology, 62, 9-17. doi:10.1080/17470210802215152

Dwyer, D. M., BoAkes, R. A., \& Hayward, A. J. (2008). Reduced palatability in lithium- and activity-based, but not in amphetamine-based, taste aversion learning. Behavioral Neuroscience, 122, 1051-1060.

Flaherty, C. F. (1996). Incentive relativity. New York: Cambridge University Press.

Forestell, C. A., \& LoLordo, V. M. (2003). Palatability shifts in taste and flavour preference conditioning. Quarterly Journal of Experimental Psychology, 56B, 140-160. doi:10.1080/02724990244000232

Grigson, P. S., Spector, A. C., \& Norgren, R. (1993). Microstructural analysis of successive negative contrast in free-feeding and deprived rats. Physiology \& Behavior, 54, 909-916.

Harris, J. A., Shand, F. L., Carroll, L. Q., \& Westbrook, R. F. (2004). Persistence of preference for a flavor presented in simultaneous compound with sucrose. Journal of Experimental Psychology: Animal Behavior Processes, 30, 177-189. doi:10.1037/0097-7403.30.3.177

HigGs, S., \& CoOPER, S. J. (1998). Evidence for early opioid modulation of licking responses to sucrose and Intralipid: A microstructural analysis in the rat. Psychopharmacology, 139, 342-355.

Holman, E. W. (1975). Immediate and delayed reinforcers for flavor preferences in rats. Learning \& Motivation, 6, 91-100.

HSIAO, S., \& FAN, R. J. (1993). Additivity of taste-specific effects of sucrose and quinine-Microstructural analysis of ingestive behavior in rats. Behavioral Neuroscience, 107, 317-326.

Myers, K. P., \& SClafani, A. (2001a). Conditioned enhancement of flavor evaluation reinforced by intragastric glucose: I. Intake acceptance and preference analysis. Physiology \& Behavior, 74, 481-493.

Myers, K. P., \& Sclafani, A. (2001b). Conditioned enhancement of flavor evaluation reinforced by intragastric glucose: II. Taste reactivity analysis. Physiology \& Behavior, 74, 495-505.

Myers, K. P., \& Sclafani, A. (2003). Conditioned acceptance and preference but not altered taste reactivity responses to bitter and sour flavors paired with intragastric glucose infusion. Physiology \& Behavior, 78, 173-183.

Sclafani, A., \& Nissenbaum, J. W. (1988). Robust conditioned flavor preference produced by intragastric starch infusions in rats. American Journal of Physiology, 255, R672-R675.

Spector, A. C., Klumpr, P. A., \& Kaplan, J. M. (1998). Analytical issues in the evaluation of food deprivation and sucrose concentration effects on the microstructure of licking behavior in the rat. Behavioral Neuroscience, 112, 678-694.

(Manuscript received April 15, 2009; accepted for publication April 24, 2009.) 